\title{
A philosophical evaluation of adaptationism as a heuristic strategy
}

\author{
Sara Green \\ Centre for Science Studies, Department of Physics and Astronomy, Aarhus University, Denmark. Email: \\ sarag@ivs.au.dk.0045 51331702
}

\begin{abstract}
Adaptationism has for decades been the topic of sophisticated debates in philosophy of biology but methodological adaptationism has not received as much attention as the empirical and explanatory issues. In addition, adaptationism has mainly been discussed in the context of evolutionary biology and not in fields such as zoophysiology and systems biology where this heuristic is also used in design analyses of physiological traits and molecular structures. This paper draws on case studies from these fields to discuss the productive and problematic aspects of this heuristic in different research practices, in functional as well as evolutionary studies on different levels of biological organization. Gould and Lewontin's Spandrels-paper famously criticized adaptationist methodology for implying the risk of generating 'blind spots' with respect to non-selective effects on evolution. Some have claimed that this bias can be accommodated through the testing of evolutionary hypotheses. Although this is an important aspect of overcoming naïve adaptationism, I argue that the issue of methodological biases is broader than the question of testability. I demonstrate the productivity of adaptationist heuristics but also discuss the deeper problematic aspects associated with the methodological imperialism that is part of the strong adaptationist position.
\end{abstract}

Keywords: methodological adaptationism, optimality, design principles, reverse engineering, design analogies, heuristic 


\section{Introduction}

The debate on adaptationism has primarily centered on questions regarding the relative causal power of selective and non-selective factors and the explanatory status of alternatives to adaptationist explanations. This paper aims to add to the literature by focusing on how adaptationism works as a heuristic, so-called methodological adaptationism (MA), and to discuss the implications of this strategy outside evolutionary biology. MA has been criticized for implying a biased perspective on evolution in assuming that biological traits are optimally designed by natural selection. Since MA is a heuristic and not an empirical claim about the biological world, the productivity is however not strictly dependent on the soundness of the assumptions made (this point will be clarified later). In fact, the bias is central for the productivity of MA because it constraints the problem space for hypothesis generation. The question I address is therefore not whether it is correct to assume that biological traits are adaptations, but to what extent this is useful or counterproductive for biologists.

I first introduce the debate on adaptationism and argue for the continued relevance of discussing MA. In introducing MA, I draw on two case studies (Section 2) from zoophysiology and systems biology. The cases will be used to contextualize philosophical distinctions in the debate on adaptationism, and to reflect on the implications of MA in functional and evolutionary analyses at different levels of biological organization. I argue that the implications of MA depend on the research context, i.e. the possibilities and requirements for testing of adaptive claims, and on what type of question it is supposed to help answer (Section 3). Furthermore, I contend that an evaluation of MA must take the dominance of MA in different research practices into account, to address aspects that go beyond the issue of testability. Finally, I argue for the relevance of a continued discussion of methodologies and testing requirements at the intersection of evolutionary biology and systems biology.

\subsection{Debating adaptationism}

Adaptationism is the study of the function and origin of biological traits in a framework where biological features are assumed to reflect the effects of natural selection. Methodological adaptationism refers to the use of such assumptions as a heuristic, often divided into two reasoning strategies called adaptive thinking and reverse engineering (Dennett 1995; Griffiths 1996). The former infers possible adaptive characters or behaviors from observed challenges to life forms in specific environments. In reverse engineering the inference goes in the opposite direction; from a biological character to an adaptive problem that the character may help solve. To guide the identification of specific relations between problems and solutions, biologists draw on knowledge from similar adaptations in other biological systems but also on analogies to good engineering design. ${ }^{1}$ Because of the intuitive appeal and successful use of this strategy, some have argued that adaptationism is not optional, but an inherent and central part of biology (Dawkins 1976; Dennett 1995). However, adaptationism has also been widely criticized.

\footnotetext{
${ }^{1}$ Design thinking is broader than adaptationism, and adaptationism can be more or less associated with design analysis (Lewens 2004). However, as the examples in this paper show, these are often connected.
} 
The most famous critique of adaptationism is Gould and Lewontin's (1979) Spandrels-paper that in a provocative tone accused the adaptationists for sharing with Voltaire's fictive Dr. Pangloss the tendency to tell just-so stories about optimal solutions. In their view, adaptationists often confuse the demonstration of current utility of characters with evidence of their origin. Furthermore, adaptationism is by Gould and Lewontin argued to nurture a reductionist picture of organisms as composed of independent traits, alienated from the environment as passive receivers of modifications by natural selection. Gould and Lewontin encourage biologists to replace naïve adaptationism with a more nuanced view of organisms as integrated wholes, and to increase the awareness of the interdependency of traits and of alternatives to selection. They illustrate the problematic inferences with an analogy to spandrels - the rounded triangular-shaped spaces formed by the intersection of arches in a cathedral. The fact that spandrels serve decorative purposes might make it tempting to conclude that this purpose explains their origin. Gould and Lewontin however see this as a mistake since spandrels are a result of architectural constraints, a necessary by-product of the decision to build a cathedral with a dome in need of stabilizing structures. ${ }^{2}$ Similarly, the historical and interdependent character of all biological traits calls for caution regarding adaptationist assumptions.

Gould and Lewontin (1979) raise two related critical points regarding i) the importance of nonselective factors in evolution, and ii) the dominance of a highly biased methodological framework. Responding to the criticism, biologists and philosophers have discussed the causal power of nonselective factors, the significance of alternative explanations and requirements for testing evolutionary hypotheses (e.g. Brandon and Rauscher 1996; Felsenstein 1985; Forber 2009; Kimura 1983; Orzack and Sober 1994a; 1994b; 1996; 2011; Sober 1996). The empirical basis for adaptive or non-adaptive claims is often taken to be the main point of Gould and Lewontin's paper. For instance, Orzack and Sober (1994b) argue that the validity of adaptationism is a matter of testing different welldefined hypotheses about the power of natural selection. In contrast, others have defended a definition of adaptationism as a heuristic (Resnik 1997). Whether defined in one way or the other, a common view is however that the criticism does not affect adaptationist heuristics but merely the falsehood of empirical assumptions about natural selection that these may rest on (Mayr 1983; Sober 1996; Resnik 1997). In contrast, Richardson views the Spandrels-paper as a challenge to adaptationist methodology understood in a broader sense and argue that the criticism goes beyond the issue of testability of adaptive claims (Richardson 2007, 54). This interpretation is supported by the fact that Gould and Lewontin's scientific work shows that they do consider historical hypotheses to be testable. Further, despite what is sometimes assumed, they do not question the causal importance of natural selection but argue that it is not the only important evolutionary factor. To clarify how the criticism goes beyond the issue of testability of adaptationist hypotheses and beyond the issue of the empirical importance of natural selection, I clarify the different definitions of adaptationism and reexamine the criticism in light of contemporary case studies.

\footnotetext{
${ }^{2}$ For a criticism of the analogy see Houston (2009).
} 


\subsection{Empirical and methodological adaptationism in different practices}

The disagreements on whether adaptationism should be understood as an empirical hypothesis or a heuristic (cf. Orzack and Sober 1996; Resnik 1997) have later been clarified by distinguishing between different kinds of adaptationism. Godfrey-Smith's (2001) describe methodological adaptationism (MA) as distinct from 'empirical adaptationism' (EA). ${ }^{3}$ EA takes into consideration the relative causal power of natural selection and is thus an empirical claim about the world. In contrast, MA is a policy recommendation for biologists on how to best approach biological systems. The different positions are often related in practice. Most biologists pursuing the adaptationist heuristic (MA) are also convinced of the power of natural selection (EA) and vice versa. The Spandrels-paper can readily be said to question the naïve forms of both types. However, as GodfreySmith points out, these different kinds do not logically entail each other. Even if it were the case that many traits are non-adaptive, biologists could still argue that to look for features of adaptation and good design is the best starting point for generating empirically testable hypotheses. The opposite is also possible although less common. Following Richardson's (2007) reading of Gould and Lewontin's work, these can be seen as exemplifying a weak form of EA (accepting the importance of natural selection) but having concerns about the reliance on MA as a general methodological framework. ${ }^{4}$ The distinction between these types of adaptationism implies that an analysis of MA is not exhausted by empirical knowledge on the relative causal role of natural selection but requires insights into how methodological adaptationism guides the formation of new biological explanations.

Problematic uses of MA are often seen in evolutionary psychology and sociobiology where adaptationist hypotheses can be constructed around any dataset (Gould 1996; Richardson 2007). Accordingly, the heuristic value of MA in these fields can be contested by pointing to the ease of the acceptance of adaptationist claims and the unwillingness to consider alternatives: "If nature disagrees with the adaptationist about what should evolve, then she has to shout. If she agrees, she has only to whisper” (Griffiths 1996, 517). In contrast, other subfields of evolutionary biology have developed strict criteria for testing historical hypotheses by drawing on phylogenetic data and functional comparisons. In this context it is therefore sometimes argued that Gould and Lewontin's criticism would kick in open doors if written today (Pigliucci 2008; Sober 1993, see however Nielsen 2009 for a different view). My aim in this paper is not to go into the debate on testing of optimality modeling in evolutionary biology but to concentrate on other research practices (zoophysiology and systems biology). I consider a better understanding of MA in these fields an important aim in its own right but discussion of MA in these fields also brings insights to the debate on adaptationism in evolutionary biology.

\footnotetext{
${ }^{3}$ Similar distinctions were made earlier (Mayr 1983; Sober 1993; Sober 1996) but the differences are most explicitly addressed in Godfrey-Smith (2001). In addition to the above mentioned types, Godfrey-Smith adds a third position called 'explanatory adaptationism' (ExA) and Lewens (2009) increase the number of types to seven. Since I shall mainly focus on MA in this paper, the distinction between MA and ExA will suffice for this paper. EA can arguably also be seen as a part of what I define as strong MA - an imperialistic account of MA that may also influence explanatory ideals in biology (Section 3 and 4).

${ }^{4}$ They would however strongly object to a form of EA undermines the importance of non-selective constraints.
} 


\section{Methodological adaptationism in different practices}

Although the key aim of zoophysiology and systems biology is to identify and demonstrate functional design, researchers from both fields often draw on adaptationist assumptions. Furthermore, systems biology exemplifies a research area where the debate on adaptationism has regained its relevance. Although systems biology is sometimes defined as a study of current biological functions only, conclusions about the origin of characters are common (Alon 2007a; Salvado et al. 2011). Because systems biologists often provide insufficient evidence for adaptive explanations, the evolutionary aspect of systems biology has been criticized for being merely a 'downstream appendix' that draws on misleading engineering analogies (Lynch 2007b; Knight and Pinney 2009). The case studies will be used to flesh out how MA can work as a productive and problematic constraint on biological reasoning, and to make philosophical distinctions on adaptationism more concrete. The first case study from zoophysiology illustrates the use of design analogies from engineering and economics and the previously mentioned distinction between reverse engineering and adaptive thinking. Furthermore, it illuminates the difference between adaptationism as a heuristic and as a hypothesis. There is no sharp distinction between these, but there is an important difference in cognitive function. A heuristic is a rule-based cognitive or representational strategy that shapes the formulation of questions and answers, often by application of guidelines from one research domain to another. In contrast, a hypothesis is a proposal about a real-world causal relation (Resnik 1997). Adaptive hypotheses can regard specific causal relations or general claims about the power of natural selection (as basis for EA). The following section shows how the use of MA, in investigations of the function of the giant nose of the sperm whale, can guide the formulation of different specific adaptive hypotheses. In Section 2.2, an example from systems biology shows the productivity of MA for discovering general network patterns. This case will however also serve as basis for the discussion of the problematic aspects of MA (Section 3 and 4).

\subsection{Case 1: What is the big nose of the sperm whale for?}

The largest nose in the world belongs to the sperm whale. In fact, the nose accounts for one third of the length of a male sperm whale and is called the spermaceti organ because it contains approximately 5000 liters of spermaceti oil. ${ }^{5}$ Assuming that organisms generally are well adapted, the hypertrophic dimension of the nose has made biologists search for an explanation of why it is so big. Biologists were also fascinated by other attributes of sperm whales, in particular their ability to deal with extreme environmental challenges. In the late 1960s, asdic recordings confirmed that sperm whales frequently dive deeper than 1000 meters to prey on giant squid (Clarke 1970). To forage in this environment whales face the challenges of i) the energy demands of deep diving and then having to surface to breathe, and ii) the difficulty of finding and catching prey in complete darkness. The following section illustrates how researchers coupled ideas about the function of the big nose (reverse engineering) to a reflection on each of these adaptive challenges (adaptive thinking). Several hypotheses on the function of the nose have been proposed but I summarize the most influential, namely; a) that its

\footnotetext{
${ }^{5}$ The name originates from early speculations by whalers that the oil could be the whale's sperm but this idea was rejected as oil was also found in females. The name however stuck with the whale (Whitehead 2003).
} 
function is to control buoyancy via thermoregulation, and b) that the nose has is used for foraging via biosonar.

\subsubsection{A buoyancy control device?}

The first scientific hypothesis on the function of the spermaceti organ was formulated by the biologist Malcolm Clarke. During a research visit in Durban in 1969, Clarke took part in field work and laboratory investigation of the density of sperm whale tissues compared to sea water under different pressures. He found that sperm whales have neutral buoyancy at surface level but must counteract positive buoyancy as they descend to forage. Based on the assumption that evolution has shaped the morphology of sperm whales to accommodate such challenges, Clarke reasoned: "Nothing about its shape suggests that it is hydrodynamically adapted for this purpose so that some control over buoyancy seems likely” (Clarke 1970, p. 873). This type of reasoning exemplifies adaptive thinking; reasoning from adaptive problems to possible solutions. A parallel analysis, initially uncoupled to the other project, regarded the functional potential of the spermaceti organ (reverse engineering). Clarke analyzed the physical and chemical properties of the spermaceti oil and found that the freezing point of the oil is $28{ }^{\circ} \mathrm{C}$. Density analyses showed that when the oil is in a liquid phase it is lighter than water and vice versa in solid phase. Clarke coupled these findings and suggested that density changes of the oil could help sperm whales control buoyancy. Scanning calorimeters were used to establish a possible comparative energy budget for diving with and without heating the oil, taking energy expenses in the biological context into account (changing water temperature and pressures, excess heat from metabolism etc.). A basic assumption was that the "energy budget" should be positive and that evolution most likely had found the most 'economic solution' (Clarke, personal communication). Accordingly, Clarke proposed that buoyancy could be adjusted via thermoregulation. Cooling the spermaceti oil with an influx of cold water until it solidifies was thus anticipated to help the whale descend, while heating the oil with excess heat from metabolism could help the whale ascend (Clarke 1970; Clarke 1978). The possibility of adjusting the temperature was supported by the finding of a dense network of capillaries in the spermaceti organ. Thus, by coupling adaptive thinking and reverse engineering, Clarke proposed that the spermaceti organ could be a heat exchanger for buoyancy control that would give sperm whales a "great selective advantage" (Clarke 1970). The proposal was published in Nature and soon became widely known, but in recent years Clarke's proposal has been criticized for lacking empirical support (Madsen 2002, Whitehead 2003). Some findings that challenge Clarke's hypotheses will be summarized in the following section.

\subsubsection{A sound generator?}

Two years after the publication of Clarke's hypothesis an alternative explanation was put forward by Kenneth Norris and George Harvey (Norris and Harvey 1972). They focused on the adaptive problem of foraging in darkness and suggested that the spermaceti organ is a sound generator used for echolocation. Sperm whales had been known to use clicks to communicate since the beginning of the last century (reviewed in Whitehead 2003), but new evidence demonstrated echolocation in other toothed whales. Due to the similar physiology of the whales' common descendants it seemed 
plausible that the spermaceti organ could play a similar role. ${ }^{6}$ It was hypothesized that the dimension of the nose meant that the sperm whale was capable of producing particularly powerful sound pressures, perhaps even sufficiently powerful to debilitate their prey (Norris and Møhl 1983). The latter extension of the hypothesis was given the nickname "the big bang theory". The hypothesis on echolocation was supported by studies of sound conduction in post mortem studies and by recordings in the field using hydrophone arrays to measure sound emission. Dissections of the spermaceti organ suggested that the organ works like an acoustic lens, and improved hydrophone arrays resulted in the recording of powerful sound pressures (Møhl 2001, Møhl et al. 2003). But the hypothesis on echolocation still faced the major challenge of demonstrating how such powerful sound pressures could be generated at great depths. According to Boyle's law, only $1 \%$ of the air inhaled at the surface is available at 1000 meters where sperm whales regularly forage and critics therefore considered this hypothesis unlikely to hold (Madsen et al. 2002).

\subsubsection{Evaluating competing adaptive hypotheses}

The controversy on the plausible function of the large nose of the sperm whale lasted several decades because it was difficult to gather empirical evidence from field research. Recordings from hydrophone arrays lacked precision and continuity because optimal recordings of clicks could only be made when the whales faced towards the array. However, a new technique for field work was developed that made simultaneous measurements of sound and depths in the field possible. Using large suction cups ( $\mathrm{T}$ on Figure 1) researchers attached depth recorders and hydrophones directly onto whales for simultaneous and precise recording of depth and click production. The recordings showed that sperm whales are able to produce very powerful clicks, even at depths below 1000 meters, because they can re-circulate the air in the nose with only a minimal diffusion of air (Madsen et al. 2002).

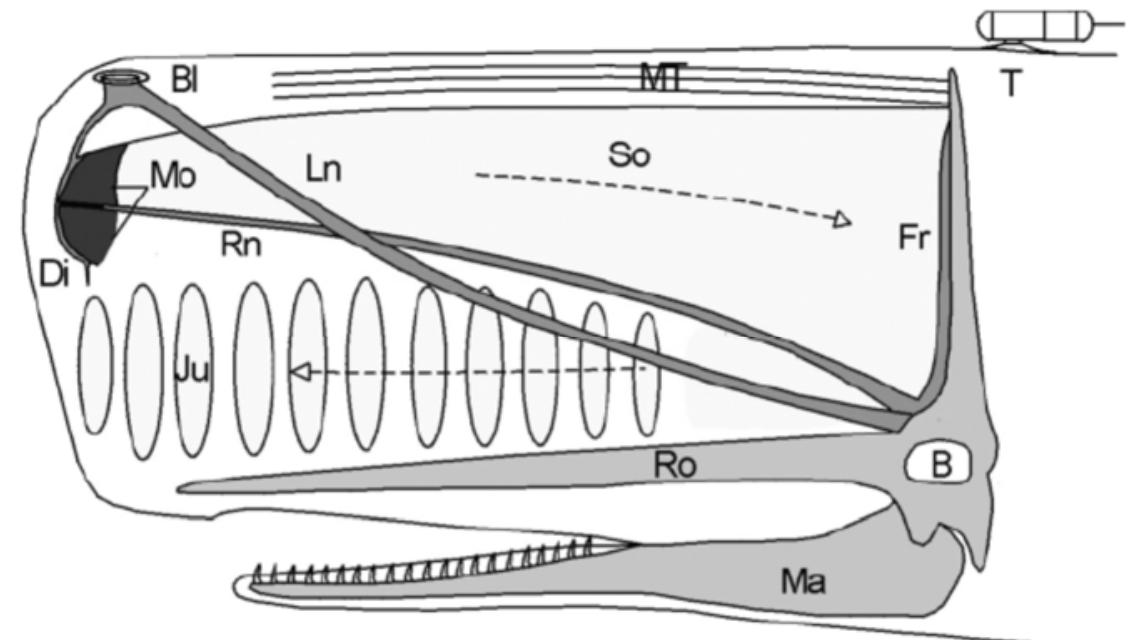

Figure 1. Schematic view of the head of a sperm whale, showing placement of a tag for recording sound (T). B: brain, BI: blow hole, Di: distal air sac, Fr: frontal air sac, Ju: junk, Ln: left naris, Ma: mandible, Mo: monkey lips, MT: muscle/tendon layer, Ro: rostrum, Rn: right naris, So: spermaceti organ. Source: Madsen et al. (2002).

\footnotetext{
${ }^{6}$ It is now known that the spermaceti organ is homologue to the right anterior bursae in phonic lips in dolphins, which is situated behind the sound-conducting organ - known as the melon - located in front of the scull.
} 
The now well-accepted mechanism for sound emission in the spermaceti organ is as follows (Figure 1); air is forced through the monkey-lips in front of the nose creating a sound pulse that passes through the spermaceti oil and is reflected in an air-filled cushion at its far end. The sound then passes through a section called the junk that redirects the sound into the water (Madsen et al. 2002; Møhl et al. 2003). The result of the investigations has been used as an argument against Clarke's hypothesis, since the velocity of sound, and thus the interval between pulses, should vary with Clarke's hypothesized temperature changes of the oil. But only a small difference in pulse interval was recorded, and this could be explained by the pressure differences at varying depths. Madsen therefore concludes that the temperature of the spermaceti oil must be constant during the dive (Madsen 2002). The result of further testing thus identified the spermaceti organ as a wide-ranging sound generator. Meanwhile, the "big-bang theory" of prey debilitation proposed by Norris and Møhl was rejected because no effect was observed when squids were exposed to high sound pressures (Wilson et al. 2008). In summary, the assumption that the spermaceti organ is well designed through selection despite its appearance focused attention on a set of possible explanations related to adaptive challenges. The controversy on the function of the spermaceti organ exemplifies how the general heuristic of adaptationism can lead to the formulation of different (here competing) adaptive hypothesis. As a result of further empirical investigations only one of these is now generally accepted. The implications of MA will be further discussed in Section 3 and compared to the following case regarding research on network motifs in systems biology.

\subsection{Case 2: Reverse engineering biological networks}

Systems biology is a relatively new field that emerged with the need for new methods to interpret and integrate data from functional genomics. Network modelling has become a widespread representational framework for investigating properties of larger systems. In the following I examine a case where overabundant network patterns were identified as generalizable design principles in regulatory networks. This work was conducted by Uri Alon's system biology group, and the publications on network motifs are currently some of the most cited papers in systems biology.

Unlike the first case study, the research on network motifs did not start with an observation of a specific structure in need of a functional explanation, but with the aim to reverse engineer unknown 'design principles’ of biological networks, abstracted from context-dependent details (Alon 2007b). A dataset on transcriptional regulatory interactions of $E$. coli was arranged as a directed graph for investigation of the networks' topological and dynamic properties (Milo et al. 2002). A guiding idea was that the topology of biological networks could resemble that of electronic networks where recurring circuit elements are used as basic design principles (Alon, personal correspondence). If so, it would be possible to find similar small overabundant patterns in biological networks and investigate their functional significance. Accordingly, Alon's group developed an algorithm to scan for sub-units in the network and compared the frequency of sub-circuits to a similar scan of randomized networks (Figure 2). 


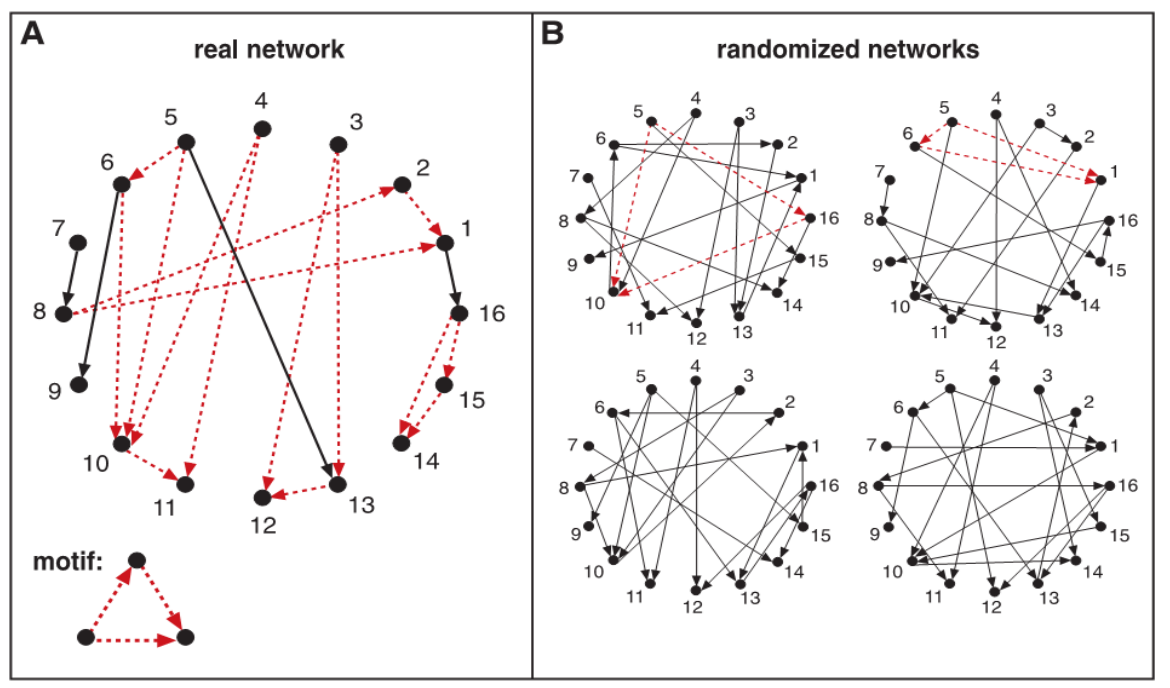

Figure 2. Sub-circuits in biological and randomized networks. Transcription factors and genes are represented as nodes. Edges (directed arrows) indicate the regulatory activity of a transcription factor on one or several operons. Dotted arrows indicate relations in motifs. Source: Milo et al. 2002.

The striking result of the initial scan was that a few patterns, called network motifs, were noticeably overabundant in the biological network. The first study of transcriptional regulation in E. coli showed three such motifs. These have also been found in eukaryotes, together with additional motifs not found in bacteria (Alon 2007b). The next step was to investigate whether different motifs have characteristic information-processing functions. The simple three-node pattern displayed on Figure 2 and 3, called a feedforward-loop (FFL), has gained special attention. It is overabundant in several species and was the first motif to be functionally investigated. From a mathematical analysis a possible function was predicted, namely that the structure could act as what in engineering is called a persistency detector for signals that activate protein synthesis (see Figure 3 for details).

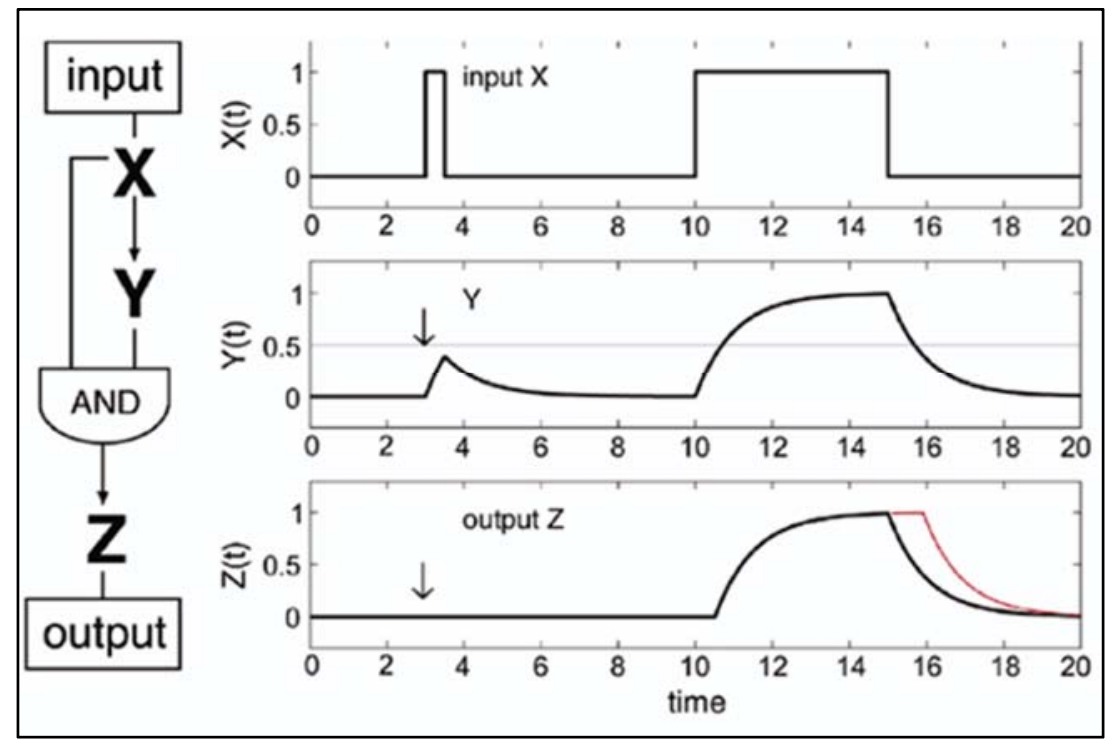

Figure 3. Activation profile of the FFL, displaying the regulatory relation as time-dependent responses to signal inputs, e.g. shifting concentrations of sugars in the environment of E. coli. X and $\mathrm{Y}$ represent transcription factors that activate a target gene $Z$. The "AND” notation signifies that activation of both $\mathrm{X}$ and $\mathrm{Y}$ is required for $\mathrm{Z}$ activation. Due to the time delay of the indirect pathway, $\mathrm{Y}$ must pass a threshold for activation by $\mathrm{X}$ that causes a delay to ON but not to OFF steps. Source: Shen-Orr et al. (2002).

Despite the simplicity of the model used, the prediction was experimentally confirmed in a setup where fluorescent reporter plasmids were incorporated in regulatory systems in E. coli to compare the activation profile for FFLs and simple circuits (Mangan et al. 2003). This positive result led to suggestions of generalizable selective benefits of specific motifs (Alon 2007a). For instance, in a noisy environment, FFL-regulation may help the cell save energy by filtering out short pulses of input 
signals that would otherwise initiate synthesis of enzymes that are not needed (Alon 2007b). These functional predictions and the overabundance of the motifs have often been taken to point to selective advantages of specific motifs. For instane, Alon contends that "[...] edges in network motifs must be constantly selected in order to survive randomization forces. This suggests that if network motifs appears in a network much more often than in randomized networks, it must have been selected based on some advantage it gives to the organism” (Alon 2007a, 29, original emphasis). ${ }^{7}$ This statement is connection to Alon's definition of 'conservative' and 'convergent' evolution that can be summarized as follows. Two genes with similar functions, stemming from a common ancestor gene, are thought to exemplify the former. 'Convergent evolution' is defined as a sign of selection acting on independent (non-homologous) traits, like the well-known example of wings for flying in bats and birds. To back up the claim about convergent evolution of network motifs, Alon refers to the lack of homology of network motifs (Alon 2007a; 2007b). Comparisons of gene sequences show that FFLs are found in different transcription factor families in yeast and bacteria (Conant and Wagner, 2003; Babu et al. 2004). Accordingly, Alon argues that if FFLs resulted from duplication of ancestral FFLs, the regulators would also be homologous.

Critics have, however, not found the divergence in orthology mapping compelling as evidence for convergent selection of network motifs, and question the background assumption of neutral evolution as a 'random process'. One criticism is that the randomized networks do not take into account preferential attachment between nodes with short spatial distance (Artzy-Randrup et al. 2004). Although this feature alone does not explain the overabundance of network motifs (Milo et al. 2004), stochastic computer simulations of network evolution with a broader notion of mutational dynamics point to the possibility that network motifs could emerge neutrally (Cordero and Hogeweg 2006; Knabe et al. 2008). Akin to Gould and Lewontin's criticism, the possibility has been raised that network motifs may be 'spandrels of genome evolution' and that the attempt to isolate regulatory patterns as functional units exemplifies a problematic atomization of traits (Solé and Valverde 2006). The problem of functional isolation refers to the high degree of overlapping nodes and edges in biological networks and to functional dependence on specific kinetic parameter values (Knabe et al. 2008; Mazurie et al. 2005). Because biological networks are complex there are heated debates as to whether the engineering analogy of design principles holds in biology and also whether too optimistic conclusions about the selective origin of network motifs have been made as a result of the guiding assumptions. Since the main focus of the paper is not to evaluate the strength of the empirical claims, I shall in the following concentrate on the possible problematic aspects of MA in light of the two cases.

\section{Reevaluating methodological adaptationism}

A common strategy to defend MA is to stress the possibilities for testing adaptive hypotheses. For instance, Resnik argues that adaptationism as a heuristic "does not have these Panglossian implications because it merely guides biological discovery, not biological justification” (Resnik 1997, 49). Although MA as a heuristic cannot be falsified (since it is not a claim about the world), the

\footnotetext{
${ }^{7}$ For similar claims see (Alon 2007b; 2007c; Shen-Orr et al. 2002).
} 
specific or general hypotheses about causal relations can (Orzack and Sober 2001). This type of argumentation has affinities with Wimsatt's (2007) more general argument that heuristics can be calibrated through triangulation of evidence. However, for Wimsatt there is no sharp separation of discovery and justification, since both are aspects of this triangulation process, and we must therefore aim for an awareness of the systematic biases of heuristics. ${ }^{8}$ Similarly, we should keep in mind that Gould and Lewontin's (1979) criticism went beyond the issue of testability in addressing criticizing how falsified adaptive claim is always replaced by another adaptive candidate. What remains unquestioned, in their view on contemporary practice, is the idea that an adaptive explanation should always be the result of analysis. The methodological and explanatory primacy of adaptationism is therefore often associated with assumptions on the power of natural selection (and thereby indirectly with EA). If it is assumed that natural selection is the only driving force of evolution, tests will not result in considerations of alternatives to adaptation. Their broader criticism therefore regards what we can call testing practices. Testing practices include methods for testing adaptive claims but also information on how such tests are used in practice for specific aims and the implications of unquestioned assumptions about the nature of biological systems.

A counterargument to this view may be that non-adaptationist methodologies can be equally biased and that if adaptationism as a research strategy is untestable, so is pluralism (Orzack and Sober 1994b). I believe this is correct. But what makes Gould and Lewontin's focus on adaptationism particularly relevant is the dominance of adaptationism. It has been debated to what extent their criticism does justice to the actual research practice back then and today (Mayr 1983; Pigliucci 2009), but what is important is that they wished to change a particular practice that they found problematic. In the following I argue that the implications of MA are sensitive to differences between research practices and to different versions of MA, addressing both strong and moderate adaptationism (see below). Orzack and Sober's other point stresses that replacing adaptationism with an equally biased null-hypothesis of neutral evolution is a problematic solution. I agree with this view. Generally, heuristics are productive because their narrow perspective constrains the space of problem solutions. The aim is therefore not to overcome the biases but to increase the awareness of these and to discuss how they may affect the end result. Wimsatt (2007) outlines four central properties of heuristics: fallibility, relative efficiency, the systematic character of their biases, and the sometimes hidden way in which they can transform problems into intuitively related but nonequivalent problems. The direction of errors for specific heuristics can be analyzed by identifying where answers to the transformed problems do not answer the original (biological) problem or where it negatively constrains the space of relevant sources. Evaluating the implications of MA therefore involves an examination of relations between the evidence required and provided for accepting adaptive claims, and a consideration of possible 'blind spots' generated by the constraints of the heuristic. These can be uncovered by considering what aspects cannot possibly be covered in a given analysis. Furthermore, evaluating heuristics requires a specification of the strength of the methodological position.

\footnotetext{
${ }^{8}$ Reichenbach's famous distinction between a context of discovery and of justification has been questioned since the 1970s, in particular in recent practice-oriented philosophical accounts (Schickore and Steinle (ed) 2009). These have argued that discovery and justification are both theoretically and temporally related.
} 
Godfrey-Smith (2001) states that adaptationist positions can come in strong and moderate versions. A moderate methodological adaptationist defends the productivity of this heuristic but does not hold that this is the only one needed in biology. In contrast, a strong account views MA as the heuristic of biology (Dawkins 1976; Dennett 1995). This account can be based on EA or on the success of previous projects where MA was used. For instance, Mayr (1983) argues that the methodology of adaptationism has been the basis of every advance in physiology. But a strong account can also be based on an argument of methodological completeness. MA may be considered the best methodology also for discovering non-selective effects because deviations from the predicted optimum in optimality modeling can be used as search tools for constraints on selection. Thus, it may be argued that MA is the only reasoning strategy biology needs even if natural selection is not the only important driving force of evolution. Based on the idea of testing practices, the strong form of MA relies on three assumptions; i) that good standards and possibilities for testing adaptive claims exist, ii) that MA does not affect the motivation to consider alternatives to selective explanations, and iii) that no other heuristic is needed because all relevant aspects can result from MA. These points are of course related but for the purpose of clarity I shall discuss these separately in the following three sections where I use the case studies to illustrate philosophical points.

\subsection{Testing requirements in different testing practices}

In the context of the adaptationism debate, attention has been drawn to testing requirements for optimality modeling. The combined use of functional predictions and phylogenetic comparisons is claimed to test for both adaptation and phylogenetic inertia and therefore to provide an unbiased testing procedure (Hansen et al. 2008). These methods are fruitful for testing adaptive claims in contexts where construction of phylogenies is possible but different testing procedures are required in different research contexts. In the following I examine the testing requirements in the cases studies and argue that these differ in functional and evolutionary studies and for different levels of analysis.

\subsubsection{Testing practices in functional analysis}

As the cases show, MA is sometimes used to guide the generation of hypotheses about the function of traits. To make sense of MA in this context, a comparison to Lewens' (2004) definition of weak adaptive thinking and weak reverse engineering is useful. The 'weaker' reasoning forms are not historical enterprises but focus on roles of parts or processes in relation to an overall capacity of systems. Since analysis does not aim to explain the origin of traits, design analogies in this context are heuristically more modest than the standard definition of MA. Although both cases examined in the previous section draw on MA, much of the research focuses on causal capacities of traits in extant organisms. Importantly, the criteria for testing such claims do not depend on evidence regarding ancestral populations (cf. Forber 2009).

In the first case on the spermaceti organ, the evidence that determined the fate of the competing hypotheses was provided by direct measurements of foraging clicks (Madsen et al. 2002). Similarly, evidence against the 'big-bang' hypothesis rested on the lack of measurable effects of powerful sound pressures on squid in the laboratory (Wilson et al. 2008). The main issue in the controversy is not an explanation for the origin of the spermaceti organ, but demonstration of the organ's capacity (in terms 
of causal mechanisms). Even when evolutionary comparisons are conducted, the primary aim is to understand how whales deal with various challenges though different designs and behavior. For instance, different species of toothed whales have been compared to investigate the relation between the size of the sound producing organ, sonar range and maximum foraging depth (Madsen, personal communication). Similarly, the evaluation of the information-processing function of network motifs is not dependent on evidence for selection, but on the match between the mathematical predictions and experimental results (Mangan et al. 2003). In both cases, the function remains unquestioned even if the trait evolved neutrally. ${ }^{9}$ The scope of functional generalizations has, in the case of network motifs, been questioned with reference to the lower frequency of network motifs relative to different null-models. However, network motifs may be functionally important regardless of their frequency. Alon's group states that overabundance compared to randomization is as a good starting point for finding functionally important patterns but he also emphasizes that the overabundant motifs are not necessarily the functionally most important ones (Shen-Orr et al. 2002; Alon, personal correspondence). It is also worth noting that recent work inspired by his approach does not base the notion of motif on frequency (Tyson and Novák 2010). Similarly, even if motifs cannot be functionally isolated in higher taxa with more dense connections, the scheme may serve as a starting point for motif analysis in more complex networks. In any case, evidence to confirm this generalization is not dependent on demonstration of convergent evolution of motifs. Thus, although phylogenetic comparisons may inform functional studies, the functional statements are not dependent on evidence for selection. In the following I discuss the implications of MA in practices geared towards conclusions about origin. I start by reflecting on MA at different levels of biological organization.

\subsubsection{MA in evolutionary analyses}

MA is often seen as particularly problematic for studies at the molecular level because the importance of neutral evolution is argued to be greater at this level (Godfrey-Smith 2001; Lynch 2007a; Kimura 1983; Koonin 2011; Pigliucci 2009). In contrast, assuming that morphological structures like the spermaceti organ are adaptations seems unproblematic. Nevertheless, the difference may not be as big at it appears. We do not have to look far from the example of the spermaceti organ to find a case where there is doubt. Unlike the sperm whale, harbor porpoises and several other toothed whales have two sets of phonic lips. It was previously thought that the additional set is an adaptation that allows porpoises to increase the power output and bandwidths of the clicks, but all recorded clicks demonstrate sound emission only from the right pair of phonic lips (Madsen et al. 2010). The historical basis for this character is still unknown, but it is possible that the explanation will involve non-selective (developmental) constraints. ${ }^{10}$ Thus, it cannot always be assumed that traits on this level have been selected as independent units. More importantly, evidence against EA is not necessarily evidence against the usefulness of MA. To evaluate MA we must examine the research context and

\footnotetext{
${ }^{9}$ I have greatly benefitted from discussions with Arnon Levy and William Bechtel regarding this issue.

${ }^{10}$ The example is analogous to Coddington’s “Two-Horn Rhinoceros Problem” (Sober 1993, 123). Even if horns in rhinos (or phonic lips in porpoises) are clear examples of adaptations, it does not follow that selection explains why some species have two instances of the trait. Similarly, the selective advantage of some network motifs does not show that the general overabundance is due to the same evolutionary mechanism.
} 
ask whether the characteristics of testing practices make it unlikely that MA influences the result of analysis.

For morphological traits, testing of adaptationist hypotheses typically involves evaluation of functional predictions (regarding relative fitness measures) and phylogenetic comparisons of the extant and ancestral character state and variation in populations (Forber 2009; Hansen et al. 2008; Orzack and Sober 2001). Evolutionary biologists generally agree on what it would take to test such hypotheses although there is an ongoing debate about how much genetic information is needed to support conclusions on historical trajectories (cf. Brandon and Raucher 1996; Pigliucci and Kaplan 2000; Pigliucci 2009). In contrast, the investigation of the origin of network patterns leaves open several questions due to the abstract nature of the topological structures investigated and the lack of knowledge about ancestral network states (Knight and Pinney 2009). The general approach for evaluation of molecular evolution is therefore often a combination of functional predictions and comparative studies to evaluate patterns of correlation for derived and conserved characters (within genomes and between groups of extant organisms). ${ }^{11}$ These methods were used in the case of network motifs, but several issues complicated the debate. First, it is often unclear whether the unit of selection refers to a FFL in a specific context or to the general type of motif (Konagurthy and Lesk 2008). Beneficial functions are often discussed as a matter of global optima (properties of FFLs in general) while evidence is established only for local optima (Mangan et al. 2003). Furthermore, the function and origin of network motifs are evaluated from different types of data sets. Proponents of the adaptive claim refer to experimental demonstration of functional significance of individual motifs in bacterial systems and anticipate a similarity in other organisms (Alon 2003; 2007a), whereas critics argue for the opposite based on computer simulations of the regulatory dynamic of more complex yeast networks (Mazurie et al. 2005; Ward and Thorton 2007). Similarly, disagreements on the degree of homology of network motifs are partly related to the different approaches used to evaluate common ancestry. One type of analysis shows no common ancestry among circuit types and considers gene duplication a rare event (Babu et al. 2004; Conant and Wagner 2003), whereas others argue for the opposite (Cordero and Hogeweg 2006; Knabe et al. 2006). However, the former type of analysis regards whole-motif duplication and target-gene duplication, whereas the latter focuses on the evolution of regulators (establishment of new binding cites between transcription factors and promoters). Some evaluate homology of genes in different species (Babu et al. 2004) whereas others focus on divergence and conservation within the same genome (Conant and Wagner, 2003; Cordero and Hogeweg 2006). Furthermore, critics have complained that the origin of network motifs is often discussed without reference to species-specific differences, neglecting the different significance of evolution via duplication and divergence in prokaryotes and eukaryotes (Kuo et al. 2006). Accordingly, the results are difficult to compare as evidence for or against general evolutionary features of network motifs. The point I want to flag is not that this issue cannot be settled or that no criteria for evaluating these accounts exits. Rather, I use the examples to show that an evaluation of MA in this context must go beyond the criteria for testing of optimality modeling and take such disagreements into consideration.

\footnotetext{
${ }^{11}$ For simple traits and short evolutionary time scales direct experimentation may however be possible, making it easier to calibrate MA with strong evidence on this level (see also Section 4).
} 


\subsubsection{Methodologies and scientific worldviews}

In Section 1 and 3 I argued that Gould and Lewontin's criticism goes beyond the issue of falsifiability. Their criticism raises a deeper philosophical question about how strictly the production and evaluation of hypotheses can be separated as independent epistemic activities. This can be illustrated by the famous controversy between Gould and Dawkins that not only reflects disagreements over the power of natural selection (EA) but also different views on how science operates (outlined in detail by Sterelny 2007). Whereas Dawkins approaches science from a falsificationist perspective, relying on requirements for testing, Gould believes that scientific knowledge is sensitive to cultural and ideological influences. Several of Gould's writings criticize research where (racist) ideological contexts have distorted the view of phenotypic differences among humans (e.g. Gould 1996). Similarly, the metaphor of the 'genetic program' has enforced a deterministic and reductionist view on the relation between genes and phenotypes. The point is not that we have no means for testing whether the genome is like a software code but that unreflected use of such 'organizing concepts' can influence how a subject matter is perceived.

Gould and Lewontin (1979) criticize the lack of interest in non-selective constraints among evolutionary biologists. They foresee a possible counterargument that has been rehearsed several times in the debate, namely that their criticism overlooks the fact that any adaptationist analysis involves considerations of drift and phylogenetic inertia. Gould and Lewontin probably painted a picture of adaptationism that is (too) easy to ridicule. Yet, they emphasized an important and longlasting difference between adaptationists and non-adaptationists on the different views on constraints. Non-adaptationists would not be satisfied with the inclusion of constraints as a source of and limit to the variation that the "real" driving force of evolution, i.e. selection, can act on. They have stressed the need to accept the constructive aspects of neutral evolution and for constraints to enter the stage of intelligible explanations (Amundson 1994). This point is echoed in the debates on adaptationism in systems biology where a central issue is the status of neutral evolution. This is not just an empirical issue but a methodological concern about the extent to which these assumptions influence the conclusions of analysis. For instance, if it is assumed that changes in the in-degree profile of networks can only result from selection, it becomes highly unlikely that other forces could explain the existence of network motifs (cf. Dekel et al. 2005; Hogeweg 2012). In other words, if neutral evolution is reduced to a randomization process, there is no point in looking for ordering principles of nonselective character, and these insights will not automatically emerge even if the adaptationist claims are met with resistance. Similarly, a recent review of methods used to discover design principles defines these as frequent functional patterns shaped by selection and remarks in a footnote that for general principles it is assumed that effects of drift and population size can be neglected (Salvado et al. 2011). This approach to design principles is considered problematic because it from the outset ignores the possibility that design principles could have a non-selective origin (Steinacher and Soyer 2012). As a counterargument it might be stated that the criticism above does not affect more advanced uses of MA that include a more adequate view of neutral evolution in testing procedures. Admittedly, the points mentioned above are not targeted at the most advanced form of MA. It does however regard 
influential contemporary research. Moreover, even if the two assumptions of strong MA remain unquestioned, it is still an issue of debate whether MA is the only heuristic needed.

\subsection{The relative efficiency of MA}

A strong version of MA would argue that adaptationist methodology is the heuristic of biology, even for discovering effects of neutral evolution. In this section I discuss this claim in relation to different aims of analysis in evolutionary (systems) biology. In particular I highlight the recent use of nonsupervised evolutionary simulations. I end the section by suggesting that these debates are also relevant for the more general issue of adaptationism in evolutionary biology. My aim is not to dispute the claim that MA can be productive for discovering non-selective factors. 'Negative results', in terms of deviations from the optimum expected, can be extremely useful in generating insights into constraints on possible trajectories. The example mentioned on the discovery of a non-functional set of phonic lips in porpoises may turn out to be one such example. It does however require that alternatives to selection are considered as serious options. Moreover, I shall argue that MA may not be the best strategy for uncovering all aspects of evolution.

MA focuses attention on features of apparent design solutions and is a productive heuristic for identifying candidates for connections between causal capacities and selective pressures. But MA appears less suited for investigating the general basis of genetic variation, structural constraints on morphology, or nonrandom effects of drift, since the research subject in these analyses is outside the scope of features of good design (Kimura 1983; Lynch 2007a; 2007b; Müller and Newman 2003). To illustrate this point I shall elaborate a little further on the case of network motifs. As we have seen, most of the research on network motifs has focused on evidence for and against adaptive origin of these patterns. A key insight in this debate however resulted from a modeling approach that did not focus on any pre-defined patterns in the first place. Cordero and Hogeweg (2006) developed a framework for studying the effects of mutational dynamics in nonsupervised network models, i.e. evolutionary simulations without a predefined goal. In this model, promoter regions were changed according to experimentally measured rates of mutational dynamics (deletion, mutation, duplication and recombination) in transcriptional regulatory networks of yeast. Although such models allow for a high degree of freedom, surprising organizational patterns sometimes appear. After 2000 generations a hierarchical structure with a scale-free distribution and overabundance of FFLconfigurations occurred (Figure 4). 
A

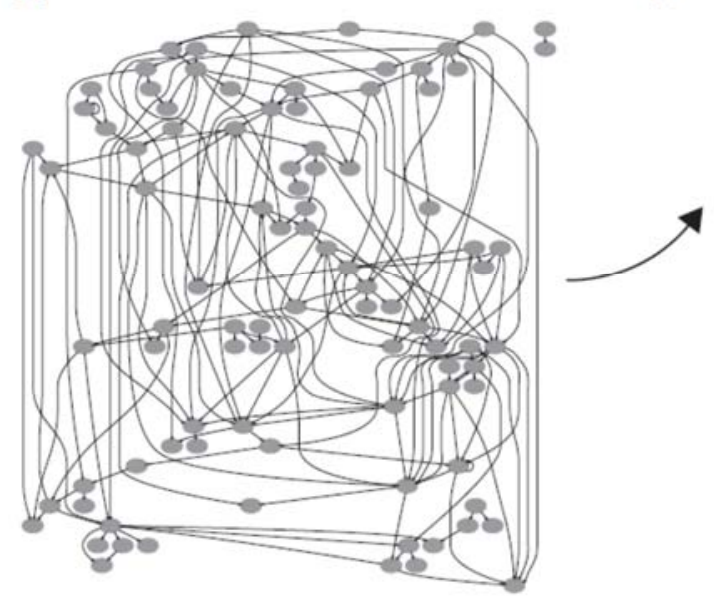

B

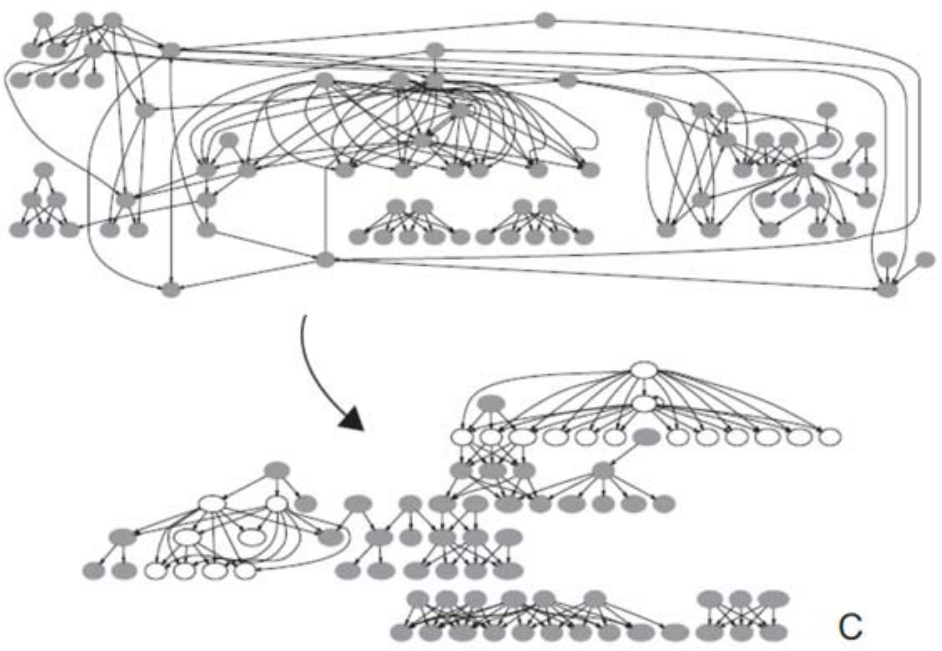

Figure 4. Generation of hierarchical network structures from non-selective tinkering of promoter regions. Nodes that take part in FFLs are marked with white. Source: Cordero and Hogeweg 2006.

Note that on the figure many of the network motifs (marked with white nodes) consist of highly overlapping network structures. At a certain point in the simulation, an avalanche of network motifs appears when a connection is added between two hub nodes (upper edge in Figure 4C). At first, the appearance of network motifs was thought to be an artifact of the model (Hogeweg, personal correspondence) but further investigations revealed that this tight overlap fits with data on network motifs in yeast networks (Cordero and Hogeweg 2006). The result does not of course demonstrate that network motifs arose due to neutral forces only. However, the simulation shows that random mutational operations can have non-random constructive effects and suggests an important role of neutral forces in the evolution of network motifs. Since the methodology is not focused on explaining the selective origin of pre-defined patterns, it is not part of adaptationist research methodology. ${ }^{12}$ The same holds for the use of mathematical tools to search for (non-selective) 'laws of evolution' in comparative studies of prokaryote and eukaryote genomes (Koonin 2011). Nevertheless, the results are directly relevant for design-oriented analyses.

So far I have mainly treated the testing requirements for network motifs as a special case where testing practices, unlike evolutionary biology, are still being developed. I end the paper by suggesting that the attempt to overcome adaptationist leanings in systems biology also will lead to reexamination of methods in evolutionary biology. While systems biology needs to integrate methods from evolutionary biology to overcome adaptationism, a new stream of evolutionary systems biologists have argued that evolutionary biology ultimately needs systems biology to go beyond statistical sequence analysis. ${ }^{13}$ As mentioned, the current methodologies for testing of adaptive hypotheses face limitations when the aim is to answer questions about characteristics of biological networks such as

\footnotetext{
12 Selective constraints can however be added to the model to identify the integrated long-term effects of mutational priming and selection.

${ }^{13}$ Evolutionary systems biology aims to integrate various fields, such as population genetics, functional genomics, developmental biology, and EvoDevo through modeling techniques from systems biology (O’Malley 2012; Jaeger and Cromback 2012).
} 
robustness, modularity and evolvability (Steinacher and Soyer 2012). This challenge has a more general scope; for global traits it is often difficult to express adaptive utility precisely enough to test the issue via a context between models (Godfrey-Smith 2001). The difficulty of drawing conclusions across specific and general contexts is further complicated by the fact that the explanatory status of natural selection may vary with the level of analysis. Wilkins and Godfrey-Smith (2009) demonstrate how different views on the role of natural selection may result if different choices are made about the level of 'grain' of adaptive landscapes. In other words, it makes a difference whether the aim is to analyze genetic variability on the molecular level, evolution of local traits on an intermediate level, or to explain the difference between the possible and realized morphospace on the highest level. Importantly, adaptationist explanations at the intermediate level are compatible with nonselective explanations at the higher level, and the limitations of MA to explain why the population exists in a specific region of the larger landscape does not counteract the productivity of the heuristic to explain local optima. But the reverse also holds; just because MA is productive for some types of analysis, it does not follow that it should be the general organizing concept of biological research. This point in fact echoes Gould and Lewontin's criticism; the problem with the (imperialistic) account of adaptationism is that it neglects the explanatory value of the lower and higher level of analysis.

Evolutionary systems biology aims to couple these different dimensions through an integration of knowledge about general principles of network evolution and specific adaptations, ideally to reach a mechanistic understanding of how particular innovations arise in evolution due to the combined effects of selection and non-selective forces. Although statistical approaches can give a measure of selective and non-selective effects, uncovering the causal details requires methods to study evolutionary processes 'directly' in vivo or in silico (Nielsen 2009; Steinacher and Soyer 2012; Wagner 2011). Researchers have begun to address these questions by combining reverse engineering methods from systems biology (network modeling of experimental data) with in silico evolution that can span larger time scales (Jaeger and Crombach 2012; O’Malley 2012). The hope is that these methodologies can provide insights not only into how molecular networks evolve but also into constraints at higher levels (Wagner 2011). It is too soon to assess the prospects of these methodologies but what I want to highlight is that evolutionary systems biology aims to extend the methodologies of evolutionary biology as well as systems biology, and to bridge the gap between neutralists and adaptationists. Nothing in this development challenges the productivity of MA. It does however challenge the methodological imperialism of strong MA.

\section{Conclusion}

Gould and Lewontin (1979) critically pictured adaptive hypotheses as just-so stories. In response to this view it has been argued that testing of adaptive hypotheses saves MA from these Panglossian implications. Furthermore, it has been argued that these testing requirements also make MA productive for discovering non-selective features. In response to this position I have emphasized that Gould and Lewontin's criticism goes beyond the issue of falsifiability. The question they raise is whether methodological habits have led to a limited view of the goal of research and the subject of study. In particular, they object to the general idea that the biologist's job is not done until an adaptive explanation is found. Modern evolutionary biology has matured since the criticism, and today new 
data and methods allow for control of both selection and phylogenetic inertia (Hansen et al. 2008; Nielsen 2009). However, testing practices are different in other research contexts.

In discussing the cases from zoophysiology and systems biology I have highlighted the different implications of MA for functional and evolutionary analysis in comparison to Lewens' (2004) description of weaker reasoning strategies. This argument does not presuppose that functional and evolutionary analysis always can be meaningfully separated. However, since the hypotheses regarding functional design do not depend on evidence for ancestral states, it is important to discuss the implications of MA in functional and evolutionary studies separately. In the debate on the origin of network motifs, I have emphasized the controversy over testing requirements. Like the Spandrelspaper, a central point of criticism is that a limited view on neutral evolution influences the result of analysis. My aim has not been to take a stand on the empirical status of network motifs but to use the case to exemplify that the characteristics of testing practices have not been settled once and for all but are continuously developing. Although the case on network motifs examined is considerably different from the use of phylogenetic comparisons, recent developments in evolutionary systems biology reveal a wish among some practitioners to extend the methodologies in evolutionary biology as well as in systems biology and to integrate insights on neutral and selective forces.

This paper does not question the idea that MA is an indispensable research tool for many research projects. Instead it aims to show that MA should not be considered the only game in town. Complementary organizing concepts and methodologies are sometimes needed to address other evolutionary questions, e.g. regarding long-term structural effects of mutational dynamics and selection. Since adaptationist and anti-adaptationist research fields (such as structuralism) have largely developed in parallel, Gould and Lewontin's criticism is still a relevant reminder of how methodological imperialism (from both sides) can hinder the integration of complementary perspectives. Perhaps because of the provocative tone in the Spandrels-paper, it is often forgotten that the paper was not just a criticism of adaptationism but a call for a methodological and explanatory pluralism. I hope this paper can serve as a reminder of the depth of the criticism and the importance of the latter point. Unfortunately, in Gould and Lewontin's paper, pluralism tends to take a back seat mainly because they chose to refer to Darwin's own pluralistic account to back up the claim; a choice that regrettably means to base the argument for pluralism on problematic subsidiary evolutionary mechanisms. As a result, Mayr (1983, 339) concludes that, "we have no choice but to fall back on the selectionist explanation”. Examining contemporary research practice suggests, however, that we do have a choice - we can benefit from the productivity of MA at the same time as being open to complementary perspectives.

\section{Acknowledgements}

I have greatly benefitted from comments from and discussions with Hanne Andersen, Sabina Leonelli, Arnon Levy, Fridolin Gross, Samuel Schindler, Maureen O’Malley and four anonymous reviewers. I would like to thank Peter Madsen, Malcolm Clarke, Paulien Hogeweg and Uri Alon for inspiration and guidance through their research. This research was possible due to funding for the 
project Philosophy of Contemporary Science in Practice, funded by the Danish Research Council for Independent Research/Humanities.

\section{References}

Alon U (2003) Biological Networks: The Tinkerer as an Engineer. Science 301:1866-1867

Alon U (2007a) An introduction to systems biology; design principles of biological circuits. Scitech Book News - LA English

Alon U (2007b) Network motifs: theory and experimental approaches. Nat Rev Genet 8:450-481

Alon U (2007c) Simplicity in biology. La nature 446:497

Amundson R (1994) Two Concepts of Constraint: Adaptationism and the Challenge from Developmental Biology. Phil of Sci 61:556-578

Artzy-Randrup Y, Fleishman SJ, Ben-Tal N, Stone L (2004) Comment on "Network Motifs: Simple Building Blocks of Complex Networks" and "Superfamilies of Evolved and Designed Networks". Science 305:1107-1107

Babu MM, Luscombe NM, Aravind L, Gerstein M, Teichmann SA (2004) Structure and evolution of transcriptional regulatory networks. Curr Opin Struct Biol 14:283-291

Brandon R, Rausher M (1996) Testing Adaptationism: A Comment on Orzack and Sober. Am Nat 148: 189-201.

Clarke M (1978) Buoyancy Control as a Function of the Spermaceti Organ in the Sperm Whale. J Mar Biol Assoc UK $58: 27-71$

Clarke M (1970) Function of the spermaceti organ of the sperm whale. Nature 28 Nov:873-874

Conant G, Wagner A (2003) Convergent evolution of gene circuits. Nat Genet 34:264-266

Cordero OX, Hogeweg P (2006) Feed-Forward Loop Circuits as a Side Effect of Genome Evolution. Mol Bio Evol 23:1931-1936

Dawkins R (1976) The Selfish Gene. Oxford University Press, Oxford.

Dekel E, Mangan S, Alon U (2005) Environmental selection of the feed-forward loop circuit in gene-regulation networks. Physical Biology 2:81

Dennett DC (1995) Darwin's dangerous idea: evolution and the meanings of life. Penguin, London

Felsenstein J (1985) Phylogenies and the Comparative Method. Am Nat 125:1-15

Forber P (2009) Spandrels and a Pervasive Problem of Evidence. Biol Philos 24:247-266

Godfrey-Smith P (2001) Three Kinds of Adaptationism. In: Orzack SH, Sober E (eds) Adaptationism and Optimality, pp 335-357

Gould SJ (1996) The Mismeasure of Man. Norton and Company, New York

Gould SJ, Lewontin RC (1979) The Spandrels of San Marco and the Panglossian Paradigm: A Critique of the Adaptationist Programme. Proc Royal Soc B 205:581-598 
Griffiths PE (1996) The historical turn in the study of adaptation. Br J Philos Sci 47:511-532

Hansen T, Pienaar J, Orzack S (2008) A Comparative Method for Studying Adaptation to a Randomly Evolving Environment. Evolution 62:1965-1977

Hogeweg P (2012) Toward a Theory of Multlevel Evolution: Long-Term Information Integration Shapes the Mutational Landscape and Enhances Evolvability. In: Soyer O (ed) Evolutionary Systems Biology. Springer, London, pp 195-223

Houston A (2009) San Marco and evolutionary biology. Biol Philos 24:215-230

Jaeger J; Crombach A (2012) Life's Attractors. Understanding Developmental Systems Through Reverse Engineering and In Silico Evolution, In: Soyer O (ed) Evolutionary Systems Biology. Springer, London, pp 93-119

Kimura M (1985) The Neutral Theory of Molecular Evolution. Cambridge University Press, Cambridge

Knabe JF, Nehaniv CL, Schilstra MJ (2008) Do motifs reflect evolved function? -No convergent evolution of genetic regulatory network subgraph topologies. BioSystems 94:68-74

Knight CG, Pinney JW (2009) Making the right connections; biological networks in the light of evolution. Bioessays 10:1080-1090

Konagurthy AS, Lesk AM (2008) On the origin of distribution patterns of motifs in biological networks. BMC Sys Bio, 2:73

Koonin EV (2011) Are There Laws of Genome Evolution? PLoS Comp Biol 7:e1002173

Kuo DP, Banzhaf W, Leier A (2006) Network topology and the evolution of dynamics in an artificial genetic regulatory network model created by whole genome duplication and divergence. BioSystems 85:177-200

Lewens T (2004) Organisms and artifacts: design in nature and elsewhere. MIT Press, Cambridge

Lewens T (2009) Seven types of adaptationism. Biol Philos 24:161-182

Lynch M (2007a) The evolution of genetic networks by non-adaptive processes. Nat Rev. Genet 8:803-813

Lynch M (2007b) The frailty of adaptive hypotheses for the origins of organismal complexity. Proc Natl Acad Sci 104:8597-8604

Madsen P (2002) Kaskelothvalens store næse. Aktuel Naturvidenskab 3:8-11

Madsen PT, Payne R, Kristiansen NU, Wahlberg M, Kerr I, Møhl B (2002) Sperm whale sound production studied with ultrasound time/depth-recording tags. J Exp Biol 205:1899-1906

Madsen PT, Wisniewska D, Beedholm K (2010) Single source sound production and dynamic beam formation in echolocating harbour porpoises (Phocoena phocoena). J Exp Biol 213:3105-3110

Mangan S, Zaslaver A, Alon U (2003) The Coherent Feedforward Loop Serves as a Sign-sensitive Delay Element in Transcription Networks. J Mol Bio 334:197-204

Mayr E (1983) How to carry out the adaptationist program? Am Nat 121:324-333

Mazurie A, Bottani S, Vergassola M (2005) An evolutionary and functional assessment of regulatory network motifs. Genome Biology 6:35.1-35.12 
Milo R, Itzkovitz S, Kashtan N, Chklovskii D, Alon U (2004) A response to Comment on "Network motifs: simple building blocks of complex networks" and "superfamilies of evolved and designed networks". Science 305:1107

Milo R, Shen-Orr S, Itzkovitz S, Kashtan N, Chklovskii D, Alon U (2002) Network Motifs: Simple Building Blocks of Complex Networks. Science 298: 824-827

Müller G, Newman SA (2003) Origination of organismal form: beyond the gene in developmental and evolutionary biology. The MIT Press, Massachusetts

Møhl B, Madsen PT, Wahlberg M, Whitlow W, Nachtigall P, Righway S (2003) Sound transmission in the spermaceti complex of a recently expired sperm whale calf. Acous Soc Am ARLO 4(1):19

Møhl B (2001) Sound transmission in the nose of the sperm whale Physeter catodon. A post mortem study. J Comp Physiol 187:335-340

Nielsen R (2009) Adaptationism - 30 years after Gould and Lewontin. Evolution 63:2487-2490

Norris KS, Harvey GW (1972) A Theory for the Function of the Spermaceti Organ of the Sperm Whale (Physeter catodon L). In: Galler et al. (eds) Animal orientation and navigation. NASA, Washington D.C., pp 397-417

Norris K, Møhl B (1983) Can Odontocetes debilitate prey with sound? Am Nat 122:85-104

O'Malley M (2012) Evolutionary Systems Biology: Historical and Philosophical Perspective on an Emerging Synthesis. Adv Exp Med Biol 751:1-28

Orzack S, Sober E (1994a) How (Not) to Test an Optimality Model. Trends Ecol Evol 9:265-267

Orzack S, Sober E (1994b) Optimality Models and the Test of Adaptationism. Am Nat 143:361-380

Orzack S, Sober E (1996) How to Formulate and Test Adaptationism. Am Nat 148:202-210

Orzack S, Sober E (2001) Adaptation, Phylogenetic Inertia, and the Method of Controlled Comparisons. In: Orzack S, Sober E (eds) Adaptationism and Optimality, Cambridge University Press, pp 45-63

Pigliucci M (2009) An Extended Synthesis for Evolutionary Biology. Ann NY Acad Sci 1168:218-228

Pigliucci M, Kaplan J (2000) The fall and rise of Dr Pangloss: adaptationism and the Spandrels paper 20 years later. Trends Ecol Evol 15:66-70

Resnik D (1997) Adaptationism: Hypothesis or Heuristic. Biol Philos 12:39-50

Richardson R (2007) Evolutionary Psychology as Maladapted Psychology. Cambridge: The MIT Press

Salvado B, Karathia H, Chimenos A, Vilaprinyo E, Omholt S, Sorribas A, Alves R (2011) Methods for and results from the study of design principles in molecular systems. Math Biosci 231:3-18

Schickore J, Steinle F (2009, eds) Revisiting Discovery and Justification, Historical and Philosophical Perspectives on the Context Distinction. Dordrecht: Springer

Shen-Orr S, Milo R, Mangan S, Alon U (2002) Network motifs in the transcriptional regulation network of Escherichia coli. Nat Genet 31:64

Sober, E (1993) Philosophy of Biology. Oxford: Oxford University Press 
Sober E (1996) Evolution and Optimality: Feathers, Bowling Balls, and the Thesis of Adaptationism. Philosophic Exchange 26:41-55

Solé RV, Valverde S (2006) Are network motifs the spandrels of cellular complexity? Trends Ecol Evol 21:419-422

Steinacher A, Soyer O (2012) Evolutionary Principles Underlying Structure and Response Dynamics of Cellular Networks. In: Soyer O (ed) Evolutionary Systems Biology. Springer, London, pp 225-247

Sterely K (2007) Dawkins vs. Gould, Survival of the Fittest. Icon Books Ltd, Cambridge

Tyson J, Novák B (2010) Functional Motifs in Biochemical Reaction Networks. Annu Rev Phys Chem 61:219-240

Wagner A (2011) The Origins of Evolutionary Innovations: A Theory of Transformative Change in Living Systems. Oxford University Press, New York

Ward JJ, Thorton JM (2007) Evolutionary Models for Formation of Network Motifs and Modularity in the Saccharomyces Transcription Factor Network. PLoS Comp Bio 3:e198

Whitehead H (2003) Sperm Whales, Social Evolution in the Ocean. The University of Chicago Press, London

Wilkins J, Godfrey-Smith P (2009) Adaptationism and the Adaptive Landscape. Biol Philos 24:199-214

Wilson M, Hanlon R, Tyack P, Madsen PT (2008) Big bang? Intense ultrasound does not have any detectable effects on the squid Loligo pealeii. Bioacoustics 17:321-323

Wimsatt WC (2007) Re-engineering philosophy for limited beings: piecewise approximations to reality. Harvard University Press, Cambridge 